\title{
Development of Perovskite-type Cobaltates and Manganates for Thermoelectric Oxide Modules
}

\author{
A. Weidenkaff', M.H. Aguirre, L. Bocher, M. Trottmann, P. Tomes, and R. Robert \\ Empa - Swiss Federal Laboratories for Materials Testing and Research Ueberlandstr.129 CH-8600 Duebendorf \\ (Received September 9, 2009; Revised October 16, 2009; Accepted December 1, 2009)
}

\begin{abstract}
Ceramics with perovskite-type structure are interesting functional materials for several energy conversion processes due to their flexible structure and a variety of properties. Prominent examples are electrode materials in fuel cells and batteries, thermoelectric converters, piezoelectrics, and photocatalysts. The very attractive physical-chemical properties of perovskite-type phases can be modified in a controlled way by changing the composition and crystallographic structure in tailor-made soft chemistry synthesis processes. Improved thermoelectric materials such as cobaltates with $p$-type conductivity and $n$-type manganates are developed by following theoretical predictions and tested to be applied in oxidic thermoelectric converters.
\end{abstract}

Key words : Thermoelectricity, Perovskite, Complex oxides

\section{Introduction}

$\mathrm{T}$ hermoelectric generators (TEGs) enable a direct heat to electricity conversion, but can nowadays only be found in very few niche applications. A much broader use of TEGs can be envisaged if new non-toxic, stable and more efficient thermoelectric materials are discovered. A broad research effort is essential to implement these very fascinating energy conversion systems in future energy technologies. The conversion efficiency of a thermoelectric converter depends strongly on the applied materials and their Figure of Merit, $\mathrm{Z}=S^{2} / \rho \kappa$. Improvements of the rather low conversion efficiency demand to find and integrate stable thermoelectric materials with ZT values $>1$ in high temperature converter modules. As of now, only TE materials present ZT values larger than unity. ${ }^{1)}$ However, improving ZT-values is not the only objective of the TE materials research. The materials need to be environmentally friendly, chemically stable at elevated temperatures, be easily available and of light weight.

Metal-oxides were identified by I. Terasaki and co-workers as promising thermoelectric materials. ${ }^{2}$ They are chemically stable even at very temperatures as high as $1200 \mathrm{~K}$. Research in this field has already led to most encouraging results for TE oxides (see Fig. 1).

Recently, the " $2 \mathrm{D}$ confinement" in titanate heterostructures $(\mathrm{ZT}=2.4$ at $600 \mathrm{~K})$ was identified as one of the most promising advances in TE research. ${ }^{10)}$

The direct conversion of the heat flux into electricity is

${ }^{\dagger}$ Corresponding author: A. Weidenkaff

E-mail : anke.weidenkaff@empa.ch

Tel : +41-44-823-4131 Fax : +41-44-823-4034 linked to electron transport phenomena, entropy per charge carrier and the interrelated Seebeck effect. With the discovery of large thermopower in metallic $\mathrm{Na}_{\mathrm{y}} \mathrm{Co}_{2} \mathrm{O}_{4}$, ${ }^{2}$ complex metal oxides have been recognized as good candidates to compete with conventional materials for thermoelectric power applications. ${ }^{13)}$ In complex transition metal oxides the spin of electrons can become an additional source of entropy resulting in the large thermopower found in these systems. ${ }^{14)}$ Complex transition metal oxides are widely used for energy conversion processes. They crystallize in characteristic coordination geometry of metal-anions polyhedra, which govern their chemical and physical properties In perovskite-type phases with the general formula $A B \mathrm{O}_{3}$, the $B$ cations form a 3D-framework of corner-sharing coordination octahedra with the anions. The slightly larger $A$ cation can

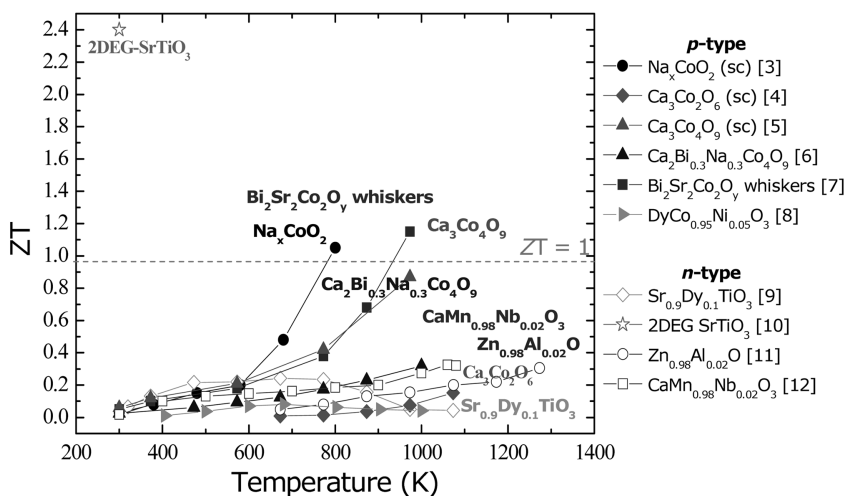

Fig. 1. ZT-values of thermoelectric oxides. Data taken from Fujita et al, ${ }^{3)}$ M. Mikami et al.,") M. Shikano et al.,5) G. Xu et al., $\left.{ }^{6}\right)$ R. Funahashi et al., ${ }^{7)}$ R. Robert et al., $\left.{ }^{8}\right)$ H. Muta et al., (9) (Results obtained under argon atmosphere), H. Ohta et $\mathrm{al}^{10)}$ et al., M. Ohtaki et al., ${ }^{11)}$ L. Bocher et al., ${ }^{12}$. 
be found inside the cub-octahedral cavity formed by eight corner sharing $\mathrm{BO}_{6}$ octahedra.

The amount of localised and mobile charge carriers and thus the electrical conductivity and thermoelectric properties in this system can be tuned by suitable substitution reactions.

The heat transfer in these complex metal oxides is governed by the lattice contribution (phonon vibrations) and the electronic component corresponding to the mobile charge carriers. For oxides the phonon contribution remains the predominant component of the total thermal conductivity. Strategies to lower the lattice heat conduction, i.e. enhance the phonon scattering include the application of nanostructures. ${ }^{15)}$ The interface scattering of phonons can dominate the heat conduction in oxide nanostructures.

In addition to cationic substitutions and introduction of defects into the oxygen sublattice, the exchange of oxygen by other anions such as halides or nitrides can be used to influence the properties of the perovskite-type compounds significantly. Therefore an oxygen- nitrogen exchange in perovskite-type oxides was performed with an ammonolysis reaction. ${ }^{16-19)}$

Promising $p$ - and $n$-type thermoelectric oxides with strongly correlated electrons are perovskite-type manganates and cobaltates studied in this work. Calcium manganates substituted with niobium are among the most promising n-type thermoelectric materials, while cobaltates are known to present a large spin-orbit entropy factor enhancing the thermopower in the system. ${ }^{20,21)}$ From the powders $p$ - and $n$-type legs were fabricated to be tested in all-oxide thermoelectric converters.

\section{Experimental}

Perovskite-type oxides with diverse composition were produced by a chimie douce synthesis process. Compacted and sintered powders of substituted cobaltates and manganates were synthesised with diverse precursor reactions. ${ }^{22,23)}$ The precursors were obtained by dissolving the required amount of soluble metal-salts in water and mixing them with a chelating agent, e.g. citric acid. These complexes were polymerised in a second step and dried to obtain homogeneous aerogel precursors. Phase purity of the products was confirmed by X-Ray Diffraction (XRD) with a PANanalytical $\mathrm{X}$ pert diffractometer using $\mathrm{Cu}-\mathrm{K} \alpha$ radiation. The morphology of the calcined powders was studied using a Scanning Electron Microscope (SEM) LEO JSM-6300F with EDX detector. The local crystal structure of the samples was further studied by Transmission Electron Microscopy (TEM) using a Philips CM 30. Rietveld refinements were performed with the FULLPROF $2000^{24)}$ software. The oxygen content of the powders was determined by a hot gas extraction method using a LECO TC 500. The transport properties measurements were performed on bar shaped pressedsintered pellets with general dimensions of $1.65 \mathrm{~mm} \times 5 \mathrm{~mm}$ $\times 1 \mathrm{~mm}$. The electrical conductivity and Seebeck coefficient

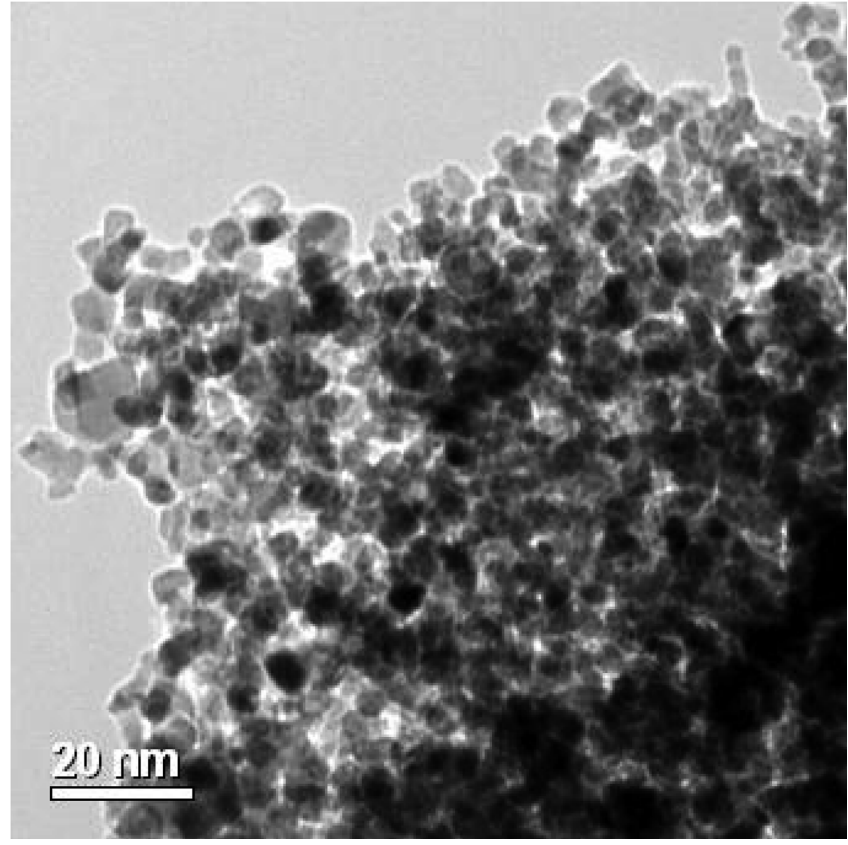

Fig. 2. TEM view of lanthanum calcium titanate particles produced by a chimie douce method.

were measured in air simultaneously as a function of temperature from $340 \mathrm{~K}$ to $1273 \mathrm{~K}$ using a RZ2001i measurement system from Ozawa Science, Japan. The electrical conductivity was determined using a four-point probe method. Two electrical contacts were positioned at both ends of the sample and the two others contacts were on the sample body. Circular pellets (10mm diameter) of the same composition were used for thermal conductivity measurements by the laser flash method using a Netzsch LFA-457 apparatus from $300 \mathrm{~K}$ to $1020 \mathrm{~K}$ in argon atmosphere. Low temperature transport and Seebeck measurements were performed with a physical property measurement system from Quantum Design.

$p$ - and $n$-type legs are produced with the obtained samples and assembled to all perovskite ceramic thermoelectric converters.

\section{Results}

A series of A and B-site substituted cobaltates, manganates, and titanates with various compositions and perovskite-type structure were successfully synthesised by a polymeric precursor method. ${ }^{23)}$ All samples are single phase and crystallize in perovskite-type crystal structures. The fine powders obtained at $\mathrm{T}<873 \mathrm{~K}$ reveal a uniform particle size of less than $50 \mathrm{~nm}$ diameter. After further annealing to $1273 \mathrm{~K}$, the sintering processes lead to an increase of particle size by a factor of 8 . The EDX and XRF analysis shows a homogeneous composition in agreement with the nominal composition. The oxygen content measurements indicate fully oxidised titanates and manganates. The cobaltates contain a slight oxygen deficiency $(\delta<0.08)$. 


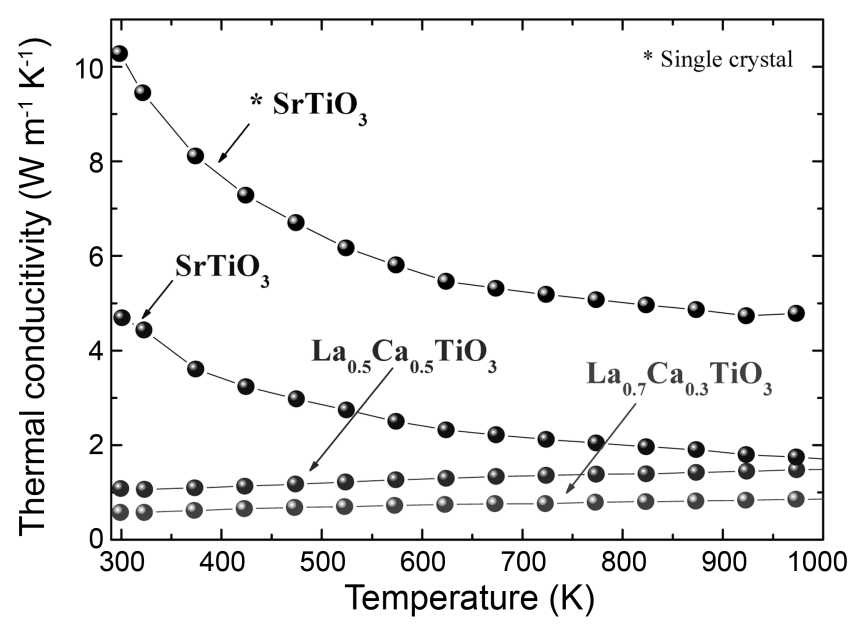

Fig. 3. Thermal conductivities of insulating titanates from different synthesis processes.

The thermal conductivity can be reduced by lowering the particle dimensions. As example the thermal conductivity of titanates with different morphologies, ranging from single crystals to polycrystalline ceramics to nanoscopic particles obtained from a chimie douce synthesis method (see Fig. 2) ${ }^{25}$ are being compared (see Fig. 3).

In chimie douce synthesis methods the crystalline size of the product particles ranges from $5 \mathrm{~nm}$ to $200 \mathrm{~nm}$ diameter. The individual crystallites are more or less agglomerated depending on the precursor and synthesis process. In ceramic solid state reaction processes the average crystallite size of the product powders typically ranges from $1 \mu \mathrm{m}$ to $10 \mu \mathrm{m}$ diameter. ${ }^{26)}$ Single crystals of perovskite-type materials can be grown in e.g. floating zone methods with diameters of up to several mm.

$\mathrm{LaCoO}_{3}$ is a promising thermoelectric material due to its high Seebeck coefficient of $600 \mu \mathrm{V} / \mathrm{K}$ at room temperature. The thermopower of $\mathrm{LaCoO}_{3}$ is positive due to the partial disproportionation $2 \mathrm{Co}^{3+} \leftrightarrow \mathrm{Co}^{2+}+\mathrm{Co}^{4+}$ and holes being the predominant mobile charge carriers. Nevertheless, the electrical resistivity is rather high $(10 \Omega \mathrm{cm})$ which lowers the thermoelectric activity and ZT values are low $\mathrm{ZT}<0.01$. The electrical resistivity generally decreases with increasing temperature, representing a semiconducting-like behaviour $(\mathrm{d} \rho / \mathrm{dT}<0)$. With aliovalent substitutions the amount of charge carriers (localized and itinerant) and thus the transport and thermoelectric properties in this system can be tuned. The electrical resistivity values of lanthanum cobaltates can be reduced with suitable Co-site and La-site substitution.

$\mathrm{Ni}$ can be introduced by substitution on the Co positions. As a result $\mathrm{LaCo}_{1-\mathrm{x}} \mathrm{Ni}_{\mathrm{x}} \mathrm{O}_{3}$ compounds are formed with improved conductivity (see Fig. 4). The activation energy of the hole hopping process of $\mathrm{LaCo}_{0.98} \mathrm{Ni}_{0.02} \mathrm{O}_{3}$ is $\mathrm{Ea}=0.089 \mathrm{eV}$, while the sample with the nominal composition of $\mathrm{LaCo}_{0.90} \mathrm{Ni}_{0.10} \mathrm{O}_{3}$ reveals an activation energy for the same process of only $\mathrm{Ea}=0.045$.

Cobalt ions $\mathrm{Co}^{3+}$ can appear in low spin-, intermediate

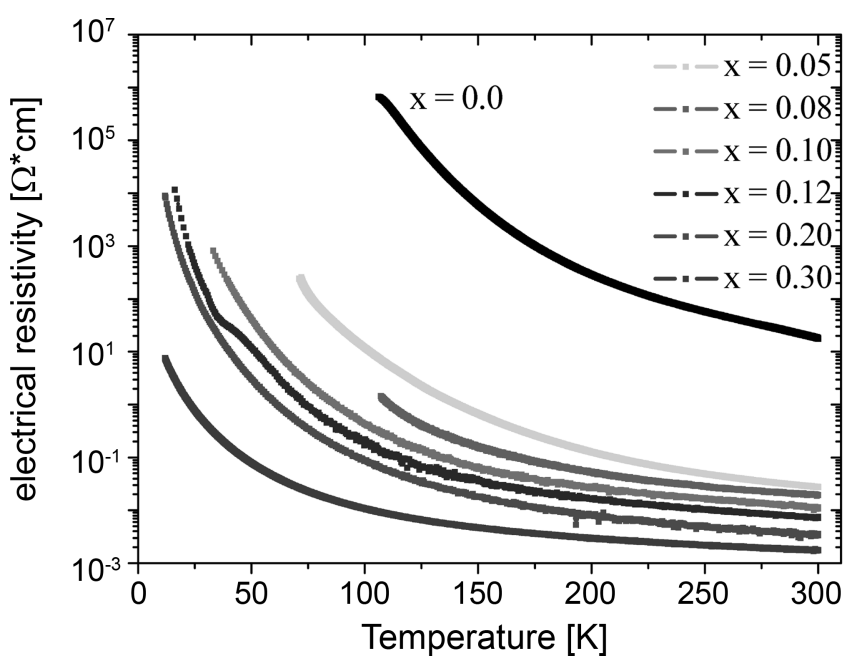

Fig. 4. Electrical resistivity of $\mathrm{LaCo}_{1-\mathrm{x}} \mathrm{Ni}_{\mathrm{x}} \mathrm{O}_{3}(0<\mathrm{x}<0.3)$ phases as function of temperature.

spin- and high spin states, leading to an additional entropy factor - the spin entropy effect. ${ }^{14)}$ This property is most probably the reason for the large thermopower found in complex cobalt oxides. The effects of the strongly interacting or "correlated" electrons in these systems are substantial and lead to exciting related properties. ${ }^{27}$ )

Lanthanum cobaltates show localised to itinerant behaviour of the d-electrons depending on the spin configuration of the cobalt ions. ${ }^{28)}$ Low spin ions are generally associated with itinerant d- electrons, while high spin ions are associated with localised electrons. ${ }^{29)}$

Important factors affecting the thermopower of complex cobalt oxides are not only the valence of the cobalt atoms, the spin of the charge carriers, the symmetry of the structure, and the strengths of the crystal field, but also the interplay of these properties. Therefore a systematic synthesis approach and in depth characterisation of the products is essential to understand the complex structure-composition-property- relations.

Generally the effect of increasing spin and orbital degrees of freedom in systems with transition metals in different oxidation states dominates the intrinsic transport and has a positive influence on the thermopower in lightly doped systems. However, with increasing substitution/carrier concentration the Coulomb interactions become less pronounced as in lightly doped systems and the effect of spin and orbital is less important. Although the thermopower is enhanced by orbital degeneracy the mechanism responsible for large thermopower are not always clear. ${ }^{30}$ )

Since changes in the crystallographic structure influence as well the transport properties, the interpretation of substitutional effects is rather complex in these systems.

$\mathrm{Ni}$ substitution causes a substantial reduction of the thermopower in La-cobaltate by a factor of $2 \sim 3$. It was further observed that the Seebeck coefficient is decreasing with rising temperature. This observation is probably due to the formation of $\mathrm{Co}^{3+}$ high spin state configuration $\left(\mathrm{t}_{2 \mathrm{~g}}{ }^{4} \mathrm{e}_{\mathrm{g}}{ }^{2}\right)$ at 
high temperatures. In $\mathrm{LaCoO}_{3}$ the $\mathrm{Co}^{3+}$ ion can be present in three different spin states. At low temperatures the $\mathrm{Co}^{3+}$ ions are in a low spin state $\left(\mathrm{t}_{2 \mathrm{~g}}{ }^{6} \mathrm{e}_{\mathrm{g}}{ }^{9}\right)$. With increasing temperature, they undergo transition to an intermediate spin state $\left(\mathrm{t}_{2 \mathrm{~g}}{ }^{5} \mathrm{e}_{\mathrm{g}}{ }^{1}\right)$ and at $1200 \mathrm{~K}$ to a high spin state configuration $\left(\mathrm{t}_{2 \mathrm{~g}}{ }^{4}\right.$ $\left.\mathrm{e}_{\mathrm{g}}^{2}\right) .{ }^{31)^{\circ}}$ The thermopower in cobalt oxides can be interpreted with the modified Heikes formula: ${ }^{32)}$

$$
\mathrm{S}=-\frac{\kappa_{\mathrm{B}}}{\mathrm{e}} \ln \left(\frac{\mathrm{g}_{3}}{\mathrm{~g}_{4}} \frac{\mathrm{x}}{1-\mathrm{x}}\right)
$$

where $x$ is the carrier concentration and $g_{3}$ and $g_{4}$ are the degeneracy of configurations of trivalent and tetravalent cobalt in the octahedral coordination, respectively. Applying this formula, large thermopower is expected for low level substituted compounds.

Thus, the absolute value of the thermopower depends on the ratio between $\mathrm{Co}^{3+}$ and $\mathrm{Co}^{4+}$ configurations and the carrier concentration. Accordingly, as Ni substitution increases the carrier concentration, a reduction of the absolute thermopower value at room temperature results in agreement with Heikes formula. ${ }^{33)}$

The decrease of the electrical resistivity with increasing $\mathrm{Ni}$ content (see Fig. 2) can be also associated to an increase of $\mathrm{Co}^{4+}$ and thus the number of holes in the mixed valance band. ${ }^{34)}$

The power factor (PF) of the Ni substituted compounds is improved, but decreases with increasing temperature.

The total thermal conductivity of the sub microcrystalline Ni-substituted lanthanum cobaltate compounds are very low $(0.34 \sim 0.48 \mathrm{~W} / \mathrm{mK})$ when compared with the reported values between $1.2 \sim 2.5 \mathrm{~W} / \mathrm{mK}$ for similar cobaltate compounds. ${ }^{35)}$

This observation can be related to the small grain size of the powders leading to a reduction of the lattice conductivity. ${ }^{36)}$ The lattice thermal conductivity is calculated using the Wiedemann-Franz-Lorenz relation. The obtained value for the lattice thermal conductivity (phononic part) corresponds to $90 \%$ of the total thermal conductivity in these systems and is decreasing with increasing $\mathrm{Ni}$ content. This leads to the assumption that the heat is carried predominantly by phonons.

The specific heat capacity of samples with composition $\mathrm{LaCo}_{1-\mathrm{x}} \mathrm{Ni}_{\mathrm{x}} \mathrm{O}_{3}(\mathrm{x}=0.05,0.08,0.15,0.20)$ is found to be independent of the temperature in the investigated region $(300 \mathrm{~K}<\mathrm{T}<1300 \mathrm{~K}$ ) ranging between 0.5 and $0.65 \mathrm{~J} / \mathrm{gK}$ and decreases with increasing $\mathrm{Ni}$ content.

The dimensionless Figure of Merit of this series of cobaltates is improved to a value of $\mathrm{ZT}=0.2$ at room temperature and remains constant with increasing temperature up to $600 \mathrm{~K}$ for samples with $\mathrm{Ni}$ contents in the range of $0.08<\mathrm{x}$ $<0.12$.

The thermal property measurements reveal similar values for the perovskite-type cobaltates and manganates in the whole temperature range from $300 \mathrm{~K}$ to $1020 \mathrm{~K}$. The measured total thermal conductivity values the cobaltates vary from $0.5 \sim 1.8 \mathrm{~W} / \mathrm{mK}$ while the manganates samples show thermal conductivities between $1.0 \mathrm{~W} / \mathrm{m} \mathrm{K}$ and $1.5 \mathrm{~W} /$

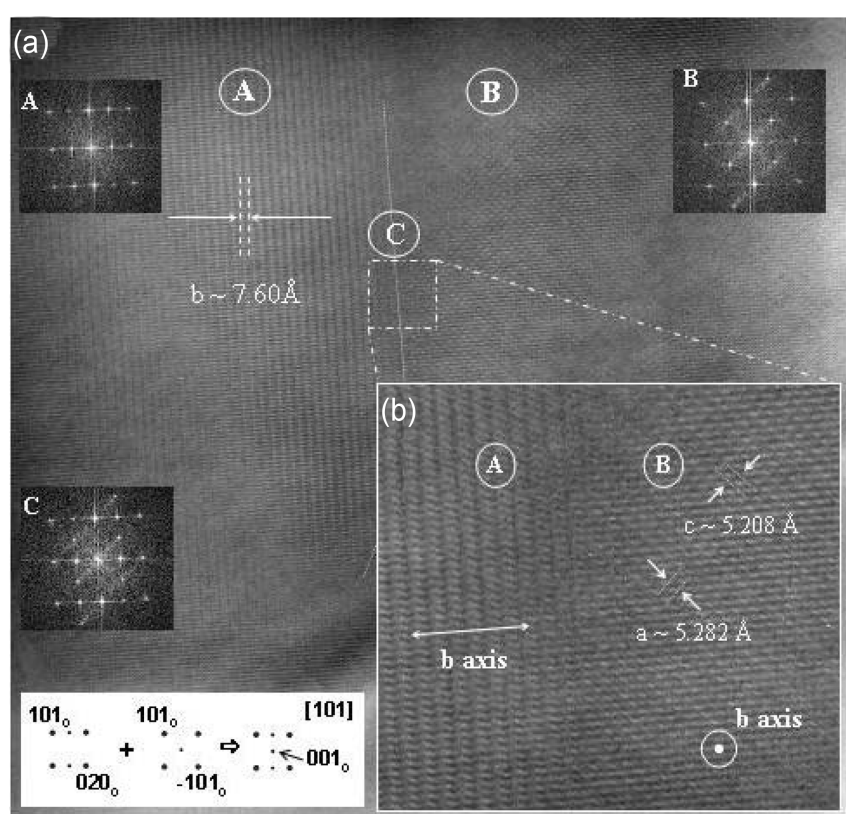

Fig. 5. (a) and (b) High-resolution TEM image of twins in the SC-derived $\mathrm{CaMn}_{0.95} \mathrm{Nb}_{0.05} \mathrm{O}_{3}$. The inset Figures present FFTs of the regions $\mathrm{A}, \mathrm{B}$ and $\mathrm{C}$.

$\mathrm{m} \mathrm{K}$ depending strongly on the particle size (see also ${ }^{34}$ ).

As $n$-type materials perovskite-type calcium manganate phases were synthesized. Polycrystalline $\mathrm{CaMn}_{1-x} \mathrm{Nb}_{x} \mathrm{O}_{3-\delta}$ phases $(x=0.02,0.05$, and 0.08) crystallize in orthorhombic symmetry with Pnma space group (S.G.) and cell parameters $\sqrt{ } 2 a_{p} \sim 2 a_{p} \sim \sqrt{ } 2 a_{p}\left(a_{p}\right.$ refers to the cubic perovskite structure). Nano-sized twinned domains characterize the microstructure of the polycrystalline manganates (Fig. 5 (a) and (b)).

Electron diffraction (ED) patterns of each domain were simulated in fast Fourier transform (FFT) mode. The FFTs from areas $\mathrm{A}$ and $\mathrm{B}$ correspond to rotation twins oriented along perpendicular $b$ axis directions: $[-101]_{0}$ and $[0-10]_{0}$. The FFT from the region $\mathrm{C}$ is the superposition of the twinned domains. Fig. 5 (b) highlights the twin direction, where the $b$ axis is either oriented in the plane (region A) or out of the plane (region B). The combination of close lattice parameters, i.e. $\mathrm{a}_{\mathrm{o}} \sim \mathrm{c}_{\mathrm{o}}$ and low octahedra tilt angles is a prerequisite for the formation of twins in orthorhombic crystal structures.

$\mathrm{CaMn}_{1-x} \mathrm{Nb}_{x} \mathrm{O}_{3-\delta}$ phases ( $\mathrm{x}=0.02,0.05$ and 0.08$)$ exhibit large absolute Seebeck coefficient and low electrical resistivity values, $\rho \sim 20 \mathrm{~m} \Omega \mathrm{cm}$ at high temperatures ( $\mathrm{T}>500 \mathrm{~K}$ ). The values correlate well with the theoretically determined values. The phases from different synthesis processes and after different thermal treatment reveal equal $S$ for the same $\mathrm{Nb}$ substitution since the thermopower depends solely on the electronic band structure (Fig. 6).

Unusually low thermal conductivity values $\left(\kappa<1 \mathrm{~W} \mathrm{~m}^{-1} \mathrm{~K}^{-1}\right)$ are achieved for the $\mathrm{CaMn}_{1-x} \mathrm{Nb}_{x} \mathrm{O}_{3-\delta}$ phases synthesized by chimie douce compared to the compounds obtained by solid state reactions, as shown in Fig. 7. Also in these compounds 


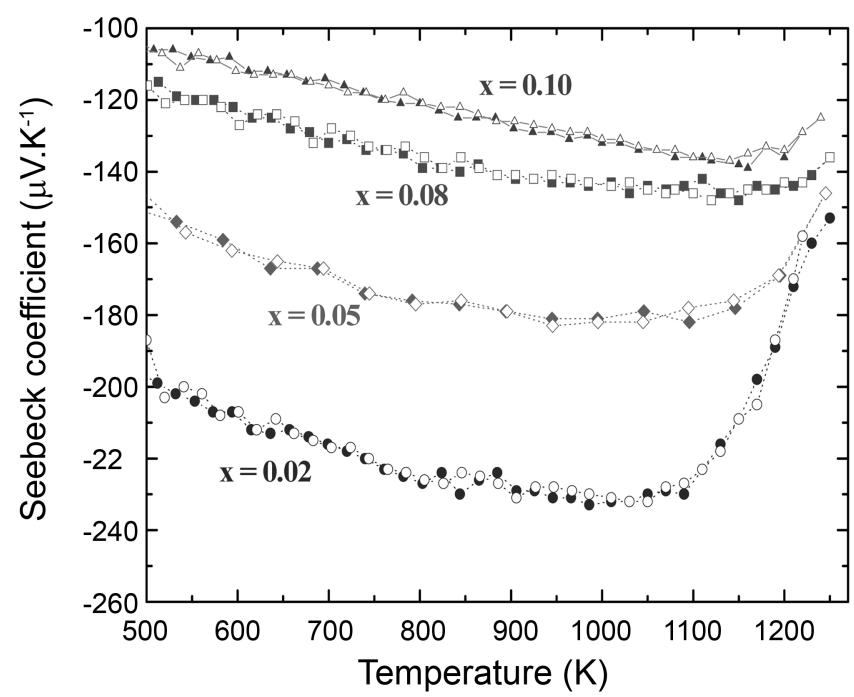

Fig. 6. Seebeck coefficient values versus $\mathrm{T}$ of $\mathrm{CaMn}_{1-\mathrm{x}} \mathrm{Nb}_{\mathrm{x}} \mathrm{O}_{3}$ of samples with te same composition (colour code) but different synthesis processes and thermal treatment (closed and half-open symbols).

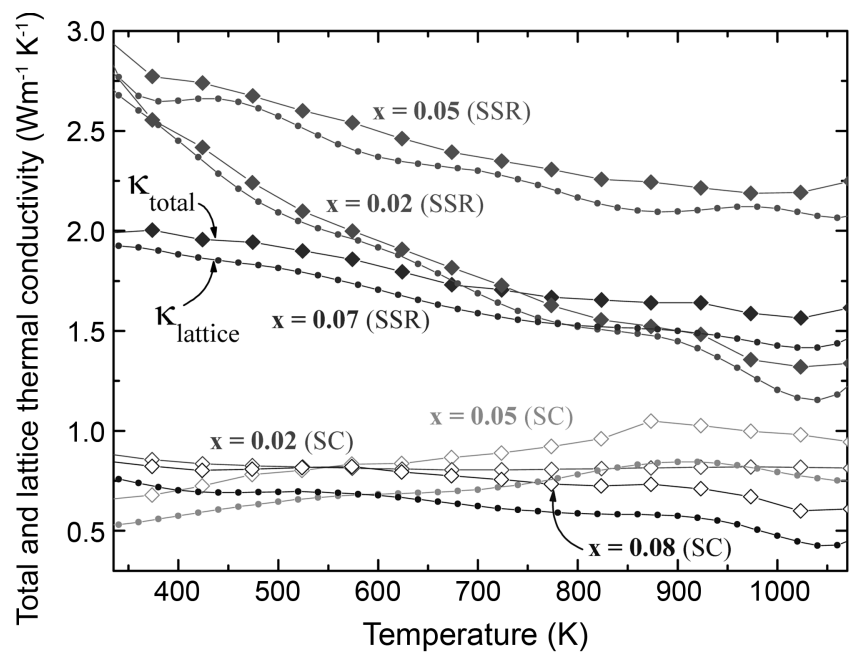

Fig. 7. Thermal conductivity versus $\mathrm{T}$ of $\mathrm{CaMn}_{1-\mathrm{x}} \mathrm{Nb}_{\mathrm{x}} \mathrm{O}_{3}$ solid state reaction- and chimie douce-series (closed and half-open symbols, respectively).

the major contribution to the thermal conductivity originates from the lattice component $\left(\kappa_{\text {lattice }}>95 \% \kappa_{\text {total }}\right)$. Introducing nano-sized twins in the chimie douce phases can act as additional scattering centers effective for the phonons but ineffective for the charge carriers. Thus, specific internal boundaries and interfaces, i.e. rotation twins, enable decreasing $\kappa$ due to phonon scattering keeping the electronic properties undisturbed. The chimie douce -series lead to $Z T=0.32$ at $1060 \mathrm{~K}$ for the $2 \% \mathrm{Nb}$-containing $\mathrm{CaMnO}_{3}$, twice as high compared to the phase obtained from solid state reactions, and represent the best perovskite-type oxides as $n$-type polycrystalline TE materials operating in air at high temperatures.

This promising $n$-type TE material is implemented in a thermoelectric oxide module together with the $p$-type

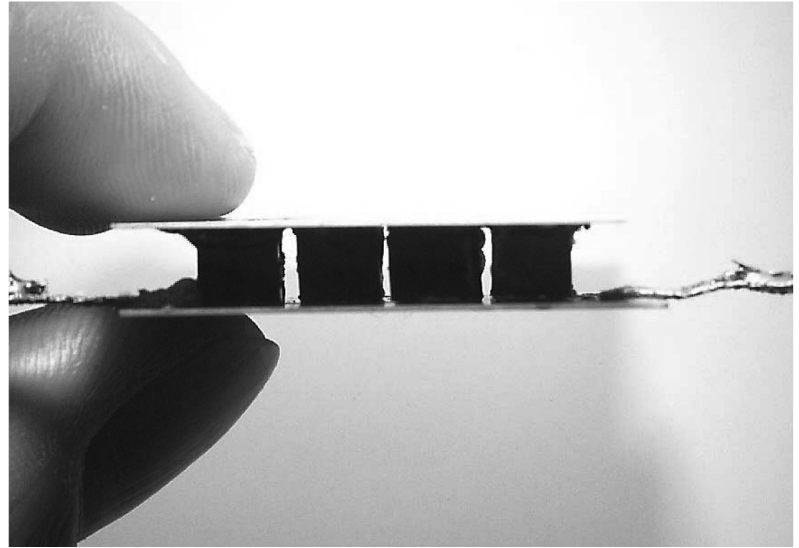

Fig. 8. photograph of a thermoelectric oxide module made of perovskite-type manganates and cobaltates as $\mathrm{p}$ - and n-type legs.

cobaltate for the direct conversion of high temperature heat into electricity.The thermoelectric materials with $p$ type conductivity as well as compounds with $n$-type conductivity have been used to produce Thermoelectric Oxide Modules (TOM) - cascades of thermoelectric oxide thermocouples- to convert the applied temperature gradient of geothermal and/or solar heat exchanger systems into electric power. ${ }^{37)}$ In these devices several ceramic $p$ - and $n$ legs are connected electrically in series and thermally in parallel to produce an open circuit voltage of several volts (see Fig. 8).

The TE converter consists of a heat exchanger, thermal and electrical connections and thermoelectric legs. The bonding process of the different ceramic components is crucial for the performance of the converter and the result of mechanical and chemical interaction between the materials. The bonding material should provide a sufficient mechanical connection and a good electrical conductivity and has to be adjusted to high temperature applications. Here materials developed for contacts in solid oxide fuel cells will be adapted.

\section{Conclusions}

Perovskite-type cobaltate and manganate thermoelectrics were successfully synthesised and characterised. The electrical conductivity, the Seebeck coefficient and the thermal conductivity were measured in a broad temperature range. The studied perovskite-type phases show potential for thermoelectric applications, as the thermopower relies on the tuneable itinerant charge carriers. The sign and the absolute value of the Seebeck coefficient can be changed with hole or electron doping.

With thermodynamical stable defects and twinned domains, reflected in the microstructure of the compounds, the thermal conductivity can be substantially lowered which is leading to improved ZT values. By combining suitable $p$ - and $n$ - type materials perovskite-type thermoelectric 
converters can be produced.

\section{Acknowledgments}

The authors thank the Swiss Federal Office of Energy for financial support.

\section{REFERENCES}

1. D.M. Rowe, "Thermoelectrics, An Environmentally-friendly Source of Electrical Power," Renewable Energy, 16 1251-56 (1999).

2. I. Terasaki, Y. Sasago, and K. Uchinokura, "Large Thermoelectric Power in $\mathrm{NaCo}_{2} \mathrm{O}_{4}$ Single Crystals," Phys.Rev. B, 56 R12685ff (1997)

3. K. Fujita, T. Mochida, and K. Nakamura, "High-Temperature Thermoelectric Properties of $\mathrm{Na}_{\mathrm{x}} \mathrm{CoO}_{2}$ Single Crystals," Japanese J. Appl. Phys., 40 4644-47 (2001).

4. M. Mikami, R. Funahashi, M. Yoshimura, Y. Morii, and T. Sasaki, "High-temperature Thermoelectric Properties of single-crystal $\mathrm{Ca}_{3} \mathrm{Co}_{2} \mathrm{O}_{6}$," J. Appl. Phys., $946579 f f$ (2003).

5. M. Shikano and R. Funahashi, "Electrical and Thermal Properties of Single-crystalline $\left(\mathrm{Ca}_{2} \mathrm{CoO}_{3}\right)_{0.7} \mathrm{CoO}_{2}$ with a $\mathrm{Ca}_{3} \mathrm{Co}_{4} \mathrm{O}_{9}$ Structure," Appl. Phys. Lett., 82 1851-53 (2003).

6. G. Xu, R. Funahashi, M. Shikano, I. Matsubara, and Y. Zhou, "Thermoelectric Properties of the Bi- and Na-substituted $\mathrm{Ca}_{3} \mathrm{Co}_{4} \mathrm{O}_{9}$ System," Appl. Phys. Lett., 80 3760-62 (2002).

7. R. Funahashi and M. Shikano, " $\mathrm{Bi}_{2} \mathrm{Sr}_{2} \mathrm{Co}_{2} \mathrm{O}_{\mathrm{y}}$ Whiskers with High Thermoelectric Figure of Merit," Appl. Phys. Lett., 81 1459-61 (2002).

8. R. Robert, M.H. Aguirre, P. Hug, A. Reller, and A. Weidenkaff, "High-temperature Thermoelectric Properties of Ln (Co, Ni) $\mathrm{O}_{3}$ (Ln = La, Pr, Nd, Sm, Gd and Dy) Compounds," Acta Materialia, 55 4965-72 (2007).

9. H. Muta, K. Kurosaki, and S. Yamanaka, "Thermoelectric Properties of Rare Earth Doped $\mathrm{SrTiO}_{3}$," J. Alloys and Compounds, 350 292-95 (2003).

10. H. Ohta, S. Kim, Y. Mune, T. Mizoguchi, K. Nomura, S. Ohta, T. Nomura, Y. Nakanishi, Y. Ikuhara, M. Hirano, H. Hosono, and K. Koumoto, "Giant Thermoelectric Seebeck Coefficient of a Two-dimensional Electron Gas in $\mathrm{SrTiO}_{3}$," Nature Materials, 6 129-34 (2007).

11. M. Ohtaki, T. Tsubota, K. Eguchi, and H. Arai, "High-temperature Thermoelectric Properties of $\left(\mathrm{Zn}_{1-\mathrm{x}} \mathrm{Al}_{\mathrm{x}}\right) \mathrm{O}$," J. Appl. Phys., 79 1816-18 (1996).

12. L. Bocher, M.H. Aguirre, D. Logvinovich, A. Shkabko, R. Robert, M. Trottmann, and A. Weidenkaff, " $\mathrm{CaMn}_{1-\mathrm{x}} \mathrm{Nb}_{\mathrm{x}} \mathrm{O}_{3}$ $(\mathrm{x}<=0.08)$ Perovskite-type Phases as Promising New Hightemperature N-type Thermoelectric Materials," Inorganic Chemistry, 47 8077-85 (2008).

13. A. Maignan, L.B. Wang, S. Hebert, D. Pelloquin, and B. Raveau, "Large Thermopower in Metallic Misfit Cobaltites," Chemistry of Materials, 14 1231-35 (2001).

14. Y. Wang, N.S. Rogado, R.J. Cava, and N.P. Ong, "Spin entropy as the Likely Source of Enhanced Thermopower in $\mathrm{NaxCo}_{2} \mathrm{O}_{4}$," Nature, 423 425-28 (2003).

15. G. Chen, G. Dresselhaus, M.S. Dresselhaus, J.P. Fleural, and T. Caillat, "Recent Developments in Thermoelectric
Materials," International Materials Reviews, 48 45-66 (2003).

16. D. Logvinovich, M.H. Aguirre, J. Hejtmanek, R. Aguiar, S.G. Ebbinghaus, A. Reller, and A. Weidenkaff, "Phase Formation, Structural and Microstructural Characterization of Novel Oxynitride-perovskites Synthesized by Thermal Ammonolysis of $(\mathrm{Ca}, \mathrm{Ba}) \mathrm{MoO}_{4}$ and $(\mathrm{Ca}, \mathrm{Ba}) \mathrm{MoO}_{3}$," J. Solid State Chem., 181 2243-49 (2008).

17. D. Logvinovich, A. Borger, M. Dobeli, S.G. Ebbinghaus, A. Reller, and A. Weidenkaff, "Synthesis and Physical Chemical Properties of Ca-substituted $\mathrm{LaTiO}_{2} \mathrm{~N}$," Progress in Solid State Chemistry, 35 281-90 (2007).

18. R. Aguiar, A. Weidenkaff, C.W. Schneider, A. Reller, and S.G. Ebbinghaus, "Synthesis and Properties of Oxynitrides $(\mathrm{La}, \mathrm{Sr}) \mathrm{Ti}(\mathrm{O}, \mathrm{N})_{3}$ Thin Films," Progress in Solid State Chemistry, 35 291-98 (2007).

19. S.G. Ebbinghaus, H.-P. Abicht, R. Dronskowski, T. Müller, A. Reller, and A. Weidenkaff, "Perovskite-related Oxynitrides - Recent Developments in Synthesis, Characterisation and Investigations of Physical Properties," Progress in Solid State Chemistry, 37 173-205 (2009).

20. R. Robert, D. Logvinovich, M.H. Aguirre, S.G. Ebbinghaus, L. Bocher, P. Tomes, and A. Weidenkaff, "Crystal Structure, Morphology and Physical Properties of LaCo1xTix $03 \pm \delta$ Perovskites Prepared by a Citric Acid Assisted Soft Chemistry Synthesis," Acta Materialia, 58 680-91 (2010).

21. A. Weidenkaff, R. Robert, M.H. Aguirre, T. Lippert, and S. Canulescu, "Development of Thermoelectric Oxides for Renewable Energy Conversion Technologies," Renewable Energy, 33 342-47 (2008).

22. E. Krupicka, A. Reller, and A. Weidenkaff, "Morphology of Nanoscaled $\mathrm{LaMO}_{3}$-particles ( $\left.\mathrm{M}=\mathrm{Mn}, \mathrm{Fe}, \mathrm{Co}, \mathrm{Ni}\right)$ Derived by Citrate Precursors in Aqueous and Alcoholic Solvents," Cryst. Eng., 5 195-202 (2002).

23. A. Weidenkaff, "Preparation and Application of Nanoscopic Perovskite Phases," Advanced Engineering Materials, 6 709-14 (2004).

24. J. Rodriguez-Carvajal, FULLPROF, p. 127, Satellite Meeting on Powder diffraction of the XVth Congress of the IUCr, 1990.

25. L. Bocher, M.H. Aguirre, R. Robert, M. Trottmann, D. Logvinovich, P. Hug, and A. Weidenkaff, "Chimie Douce Synthesis and Thermochemical Characterization of Mesoporous Perovskite-type Titanate Phases," Thermochim. Acta, 457 11-9 (2007).

26. A. Weidenkaff, R. Robert, M.H. Aguirre, L. Bocher, and L. Schlapbach, "Nanostructured Thermoelectric Oxides with Low Thermal Conductivity," Phys. Status Solidi., 6247 (2007).

27. G. Kotliar and D. Vollhardt, "Strongly Correlated Materials: Insights from Dynamical Mean-Field Theory," Physics Today, 53-9 (2004).

28. P.M. Raccah and J.B. Goodenough, "First-Order LocalizedElectron <-> Collective Electron Transition in $\mathrm{LaCoO}_{3}$," Phys.Rev. B, 155 932-43 (1967).

29. C.N.R. Rao, O. Parkash, and P. Ganguly, "Electronic and Magnetic Properties of $\mathrm{LaNi}_{1 \cdot \mathrm{x}} \mathrm{Co}_{\mathrm{x}} \mathrm{O}_{3}, \mathrm{LaCo}_{1-\mathrm{x}} \mathrm{Fe}_{\mathrm{x}} \mathrm{O}_{3}$ and $\mathrm{LaNi}_{1-\mathrm{x}} \mathrm{Fe}_{\mathrm{x}} \mathrm{O}_{3}$, J. of Solid State Chemistry, 15 186-92 (1975). 
30. W. Koshibae and S. Maekawa, "Effect of Spin and Orbital On Thermopower in Strongly Correlated Electron Systems," J. of Magnetism and Magnetic Materials, 258-59 216-18 (2003).

31. M.A. Senaris-Rodriguez and J.B. Goodenough, "Magnetic and Transport Properties of the System $\mathrm{La}_{1-\mathrm{x}} \mathrm{Sr}_{\mathrm{x}} \mathrm{CoO}_{3-\delta}$ $(0<\mathrm{x}<0.50)$," J. Solid State Chemistry, 118 323-36 (1995).

32. W. Koshibae, K. Tsutsui, and S. Maekawa, "Thermopower in Cobalt Oxides," Phys.Rev. B, 62 6869-72 (2000).

33. R.R. Heikes, R.C. Miller, and R. Mazelsky, "Magnetic and Electrical Anomalies in $\mathrm{LaCoO}_{3}$, "Physica B: Condensed Matter, 30 1600-8 (1964).

34. L. Bocher, R. Robert, M.H. Aguirre, L. Schlapbach, and A.
Weidenkaff, "Thermoelectric Perovskite-Type Oxides for Geothermal and Solar Energy Conversion," Proc. of the 4th European Conference on Thermoelectrics, 143 (2006).

35. P. Migiakis, J. Androulakis, and J. Giapintzakis, "ThermoElectric Properties of $\mathrm{LaNi}_{1-\mathrm{x}} \mathrm{Co}_{\mathrm{x}} \mathrm{O}_{3}$ Solid Solution," J. Appl. Phys., 94 7616-20 (2005).

36. G. Chen, "Phonon Heat Conduction in Nanostructures," International J. Thermal Sciences, 39 471-80 (2000).

37. P. Tomes, R. Robert, M. Trottmann, L. Bocher, M. Aguirre, J. Hejtmanek, and A. Weidenkaff, "-type Thermoelectric Oxide Modules (TOM) for Electric Power Generation," JEM submitted (2009). 